\title{
Voltages Across an Area of a Network
}

\author{
Ian Dobson, Fellow, IEEE
}

\begin{abstract}
We define the voltage across an area of a resistive network by suitably combining voltages at buses (nodes) on the border of the area. The new voltage across the area satisfies circuit laws. The same concept works to define the voltage angle difference across an area of a DC load flow network and the complex voltage difference across an area of an AC load flow network. We first define the voltage across a cutset of lines, and then derive and explain the voltages across areas, including generalizations to several voltages across an area and multiple areas. The new voltages across areas seem promising for both power system monitoring and network reduction, and we describe their application to monitoring area stress.
\end{abstract}

Index Terms-Circuit analysis, network theory, phasor measurement units, power grids, power system modeling, smart grids.

\section{INTRODUCTION}

$\mathbf{T}$ HE difference of voltages at two buses or nodes is fundamental to power system engineering. For example, bus voltages are the difference between voltage at a bus and the voltage at a reference bus, and the voltage across a transmission line is the difference between the voltages at the buses at each end of the line. The goal of this paper is to introduce new concepts of voltage differences between sets of buses. First we define the voltage difference between sets of buses separated by a cutset of lines and then we show how to extend this definition to voltages across an area of a power system. We also define area conductance and the current through the area in a natural way. The new electrical quantities for the area satisfy circuit laws and are expected to be useful in monitoring, analyzing, and reducing power transmission networks.

We develop the voltages across cutsets or areas starting from the basic ingredients of electrical circuits: a network, a variable "across" the transmission lines such as voltage, a variable "through" the lines such as current, an "admittance-like" property of the lines such as conductance, and an "Ohm's law" relating these quantities [1]. Except for notation, the development is the same for any choice of the across, through, and admittance-like quantities. Table I shows three useful choices of these quantities for electric power engineering. Since the resistive circuit is the most basic electrical circuit, the paper develops the cutset and area voltages using the resistive circuit notation, but

Manuscript received May 21, 2011; revised August 06, 2011 and September 08, 2011; accepted September 09, 2011. Date of publication November 10, 2011; date of current version April 18, 2012. The work described in this paper was coordinated by the Consortium for Electric Reliability Technology Solutions with funding provided in part by the California Energy Commission, Public Interest Energy Research Program, under Work for Others Contract No. 500-99-013, BO-99-2006-P. The Lawrence Berkeley National Laboratory is operated under U.S. DOE Contract No.DE-AC02-05CH11231. This work was supported in part by the US DOE project "The Future Grid to Enable Sustainable Energy Systems," an initiative of PSERC. Paper no. TPWRS-00478-2011.

The author is with the Electrical and Computer Engineering Department, Iowa State University, Ames, IA 50011 USA (e-mail: dobson@ iastate.edu).

Digital Object Identifier 10.1109/TPWRS.2011.2168985

\begin{tabular}{cllll}
\multicolumn{5}{c}{ TABLE I } \\
CIRCUIT QuANTITIES \\
circuit type & $\begin{array}{c}\text { "across" } \\
\text { variable }\end{array}$ & $\begin{array}{c}\text { "through" } \\
\text { variable }\end{array}$ & "admittance" & "Ohm's law" \\
\hline Resistive & voltage $V$ & current $I$ & conductance $g$ & $I=g V$ \\
DC load flow & angle $\theta$ & power $P$ & susceptance $b$ & $P=b \theta$ \\
AC load flow & voltage $V$ & current $I$ & admittance $y$ & $I=y V$ \\
& all AC load flow circuit quantities are complex
\end{tabular}

it is trivial to substitute the appropriate notation to obtain the corresponding results for the DC and AC load flow networks.

The applications of area voltages in power systems include model reduction and synchrophasor monitoring of area stress and line trips [2]-[4]. The concept of voltage phasor angle across a cutset of lines seems to be first introduced in [2]. The cutset voltage angle is extended to measure voltage angles across "cutset areas" in [3]. Cutset areas are special areas that separate the other areas of the power system from each other. The voltage angles across cutset areas can be monitored by synchrophasor measurements at the border buses and used to monitor cutset area stress and whether line trips occur in the cutset area [3]. The conference paper [4] defines voltage angles across more general areas and describes their application to monitor area stress and to detect line trips. In this paper, motivated by these applications, we completely rework and generalize the theory of voltages across power system areas, and describe their application to monitor area stress.

\section{Voltage ACROSS A CUTSET}

\section{A. Example of Cutset Voltage}

Consider the example resistive network on the left-hand side of Fig. 1. There are current injections at the buses (nodes) that cause current flows and voltage differences in the network. The network buses $M=\{1,2,3,4,5\}$ are partitioned into two subsets of buses $M_{a}=\{1,2,3\}$ and $M_{b}=\{4,5\}$. The buses in $M_{a}$ are separated from the buses in $M_{b}$ by the cutset of lines $1,2,3,4,5,6$ that is indicated by the dashed line. (Recall from circuit theory that a cutset of lines is a set of lines that cuts the network into separate networks when that set of lines is removed from the network. ${ }^{1}$ ) The objective is to define the voltage $\hat{V}_{a b}$ across the cutset from the buses in $M_{a}$ to the buses in $M_{b}$ that satisfies Ohm's law.

Write $V_{i}$ for the voltage at bus number $i$ and $\hat{V}_{j}$ for the voltage difference across line number $j$. The conductance of line number $j$ is $g_{j}$. The current flowing from $M_{a}$ to $M_{b}$ along line $j$ of the cutset is $g_{j} \hat{V}_{j}$. For this example, it is convenient to assume that the voltage difference $\hat{V}_{j}$ on line $j$ is positive for positive current flowing on line $j$ from $M_{a}$ to $M_{b}$. The current

\footnotetext{
${ }^{1}$ Some authors define a cutset to be a minimal set of buses or lines that separate the network, but we do not require this here.
} 


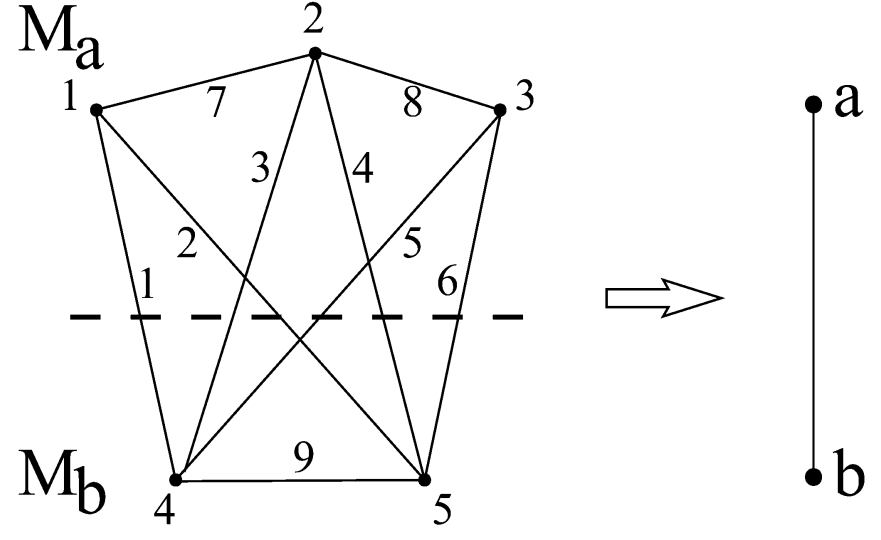

Fig. 1. Cutset voltage example: Buses 1,2,3 in $M_{a}$ are separated from buses 4,5 in $M_{b}$ by the cutset of lines $1,2,3,4,5,6$. The cutset is indicated by the dashed line. The five-bus network is contracted to an equivalent single line in which $M_{a}$ is contracted to bus $a$ and $M_{b}$ is contracted to bus $b$.

$I_{a b}$ flowing through the cutset from $M_{a}$ to $M_{b}$ is the sum of the currents flowing in each line of the cutset:

$$
I_{a b}=g_{1} \hat{V}_{1}+g_{2} \hat{V}_{2}+g_{3} \hat{V}_{3}+g_{4} \hat{V}_{4}+g_{5} \hat{V}_{5}+g_{6} \hat{V}_{6} .
$$

The cutset conductance is defined to be

$$
g_{a b}=g_{1}+g_{2}+g_{3}+g_{4}+g_{5}+g_{6} .
$$

This definition of cutset conductance is expected because the cutset lines are in parallel. We define the voltage across the cutset from $M_{a}$ to $M_{b}$ as

$$
\hat{V}_{a b}=\frac{g_{1}}{g_{a b}} \hat{V}_{1}+\frac{g_{2}}{g_{a b}} \hat{V}_{2}+\frac{g_{3}}{g_{a b}} \hat{V}_{3}+\frac{g_{4}}{g_{a b}} \hat{V}_{4}+\frac{g_{5}}{g_{a b}} \hat{V}_{5}+\frac{g_{6}}{g_{a b}} \hat{V}_{6}
$$

which is a linear combination of the cutset line voltage differences, weighted according to the line conductances. Then (1), (2), and (3) imply Ohm's law for the cutset $I_{a b}=g_{a b} \hat{V}_{a b}$.

Equation (3) can be rewritten in terms of the bus voltages $V_{1}, V_{2}, V_{3}, V_{4}, V_{5}$ as

$$
\begin{aligned}
\hat{V}_{a b}=\frac{g_{1}+g_{2}}{g_{a b}} V_{1}+ & \frac{g_{3}+g_{4}}{g_{a b}} V_{2}+\frac{g_{5}+g_{6}}{g_{a b}} V_{3} \\
& -\frac{g_{1}+g_{3}+g_{5}}{g_{a b}} V_{4}-\frac{g_{2}+g_{4}+g_{6}}{g_{a b}} V_{5} .
\end{aligned}
$$

This example shows how a cutset voltage can be defined to be consistent with Ohm's law. Defining the voltage, current and conductance of the cutset can be regarded as contracting the network ${ }^{2}$ to a single line equivalent joining two buses as illustrated in Fig. 1.

\section{B. General Case of Cutset Voltage}

More generally, consider a connected resistive network in which the buses $M$ of the network are partitioned into two subsets $M_{a}$ and $M_{b}$. Let $c$ be the cutset of lines in the subnetwork

\footnotetext{
${ }^{2}$ This operation is called vertex contraction in graph theory.
}

that separates $M_{a}$ and $M_{b}$. Define the lines in the cutset with the row vector $c$, where

$$
c_{j}= \begin{cases}1 & \text { line } j \text { in cutset has sending end in } M_{a} \\ -1 & \text { line } j \text { in cutset has receiving end in } M_{a} \\ 0 & \text { line } j \text { not in cutset. }\end{cases}
$$

The line conductances $g_{1}, g_{2}, \ldots, g_{\text {nline }}$ are assumed to be positive. The diagonal matrix of line conductances is

$$
\Lambda=\operatorname{diag}\left\{g_{1}, g_{2}, \ldots, g_{\text {nline }}\right\} .
$$

Write the vector of voltages across the lines as $\hat{V}$. Then the current flow through the cutset from $M_{a}$ to $M_{b}$ is

$$
I_{a b}=\sum_{j} c_{j} g_{j} \hat{V}_{j}=c \Lambda \hat{V} .
$$

Define the conductance of the cutset

$$
g_{a b}=\sum_{j} g_{j}=c \Lambda c^{T} .
$$

The cutset conductance $g_{a b}$ is positive since the line conductances are positive and the connectedness of the network implies that $c$ is not zero. One consequence of (6) is that if a line is not in the cutset, its conductance is not included in the cutset conductance.

Define the voltage across the cutset from $M_{a}$ to $M_{b}$ as

$$
\hat{V}_{a b}=\frac{c \Lambda \hat{V}}{g_{a b}} .
$$

Then (5), (6), and (7) imply Ohm's law for the cutset

$$
I_{a b}=g_{a b} \hat{V}_{a b} \text {. }
$$

Now we express the formulas for cutset voltage, conductance, and current in terms of the bus voltages. Let $V_{m}$ be the column vector of bus voltages. $A$ is the incidence matrix

$$
A_{i j}= \begin{cases}1 & \text { bus } i \text { is sending end of line } j \\ -1 & \text { bus } i \text { is receiving end of line } j \\ 0 & \text { otherwise }\end{cases}
$$

that relates bus voltages $V_{m}$ to the line voltage differences $\hat{V}$ :

$$
\hat{V}=A^{T} V_{m} \text {. }
$$

The row vector $\sigma_{a}$ defines the buses in $M_{a}$ by

$$
\left(\sigma_{a}\right)_{i}= \begin{cases}1 & \text { bus } i \text { in } M_{a} \\ 0 & \text { otherwise. }\end{cases}
$$

Then the lines in the cutset are given by

$$
c=\sigma_{a} A
$$

and the network conductance matrix is ${ }^{3}$

$$
G_{m m}^{\mathrm{red}}=A \Lambda A^{T} \text {. }
$$

${ }^{3}$ The superscript red stands for "reduced" and is included in the notation for compatibility with Section III. The network considered in this section becomes a Kron-reduced subnetwork of border buses $M$ in Section III. 
The conductance matrix $G_{m m}^{\text {red }}$ is symmetric and its rows add to zero. Moreover, since the network is assumed to be connected, $G_{m m}^{\text {red }}$ is irreducible. Now, using (11), the cutset conductance $g_{a b}$ defined in (6) is re-expressed as

$$
g_{a b}=\sigma_{a} G_{m m}^{\mathrm{red}} \sigma_{a}^{T}
$$

and the cutset voltage $\hat{V}_{a b}$ defined in (7) is re-expressed as

$$
\hat{V}_{a b}=\frac{\sigma_{a} G_{m m}^{\mathrm{red}} V_{m}}{g_{a b}} .
$$

Moreover, if we write $I_{m}^{\text {red }}$ for the column vector of bus current injections, then

$$
I_{a b}=\sigma_{a} I_{m}^{\mathrm{red}}
$$

is both the total current injected in buses $M_{a}$ and the current that flows from $M_{a}$ to $M_{b}$ through the cutset $c$.

The Ohm's law equations for the network are

$$
I_{m}^{\mathrm{red}}=G_{m m}^{\mathrm{red}} V_{m}
$$

The Ohm's law (8) for the cutset can now be derived in a direct way by multiplying (16) by $\sigma_{a}$ :

$$
I_{a b}=\sigma_{a} I_{m}^{\mathrm{red}}=\sigma_{a} G_{m m}^{\mathrm{red}} V_{m}=g_{a b} \frac{\sigma_{a} G_{m m}^{\mathrm{red}} V_{m}}{g_{a b}}=g_{a b} \hat{V}_{a b} .
$$

It is possible that some buses in $M_{a}$ do not have any incident lines in the cutset $c$. The entries of the row vector $\sigma_{a} G_{m m}^{\text {red }}$ corresponding to these buses are zero 4 and it follows that the voltages of these buses do not appear in the formula (7) for the cutset voltage. This also holds for buses in $M_{b}$ that do not have any incident lines in the cutset $c$.

The cutset voltage can also be derived as a novel choice of coordinates in classical network theory. This is explained in Appendix A.

\section{VOLTAGE ACROSS AN AREA}

We define the voltage $\hat{V}_{a b}$ between a set of buses $M_{a}$ and a set of buses $M_{b}$ and across an area $\mathrm{R}$ of a resistive network. We also define the current flow $I_{a b}$ through area R from $M_{a}$ to $M_{b}$ and the conductance $g_{a b}$ of area R from $M_{a}$ to $M_{b}$. The sets of buses $M_{a}$ and $M_{b}$ are assumed to be nonempty and have no buses in common. We write $M=M_{a} \cup M_{b}$ for the buses in $M_{a}$ or $M_{b}$. The buses $M$ in this section correspond to all of the buses of the network considered in Section II.

The area $\mathrm{R}$ is a subnetwork that we assume to be connected. The buses in $\mathrm{R}$ are partitioned into border buses $M$ and interior buses $N$. The interior buses $N$ must have no incident lines joining them to buses outside R. ${ }^{5}$ Each border bus in $M$ can have incident lines joining it to buses outside $\mathrm{R}$, but this is not required. Fig. 2 shows an example of an area $\mathrm{R}$ in the 39-bus New England test system. There are usually multiple ways to divide the border buses $M$ into the buses $M_{a}$ and $M_{b}$. For example, in Fig. 2, we can choose $M_{a}=\{31,32\}$ and $M_{b}=\{39,4,14\}$,

\footnotetext{
${ }^{4}$ A column $w$ of $G_{m m}^{\text {red }}$ corresponding to buses in $M_{a}$ with no incident lines in the cutset $c$ has zeros in the rows corresponding to $M_{b}$. Therefore, $\sigma_{a} w$ is the sum of all the nonzero entries of $w$ and $\sigma_{a} w=0$ since $1 G_{\mathrm{mm}}^{\mathrm{red}}=0$.

${ }^{5}$ This section assumes that there is at least one interior bus since Section II treats the case of no interior buses.
}

or we can choose $M_{a}=\{31,32,39\}$ and $M_{b}=\{4,14\}$. Each choice of $M_{a}$ and $M_{b}$ gives different quantities $V_{a b}, I_{a b}$, and $g_{a b}$. That is, the electrical properties from one side of an area to the other side of the area depend on how the "sides" are chosen.

Recall from circuit theory that a nodal cutset of buses is a set of buses that cuts the network into separate networks when that set of buses is removed from the network. It is usual and useful to choose $M$ to be a nodal cutset. If $M$ is a nodal cutset, then the network that remains when the buses in $M$ are removed from the network has at least two components. One or several of these components can be chosen as the interior buses $N$. However, to avoid buses in $N$ being directly connected to buses outside $\mathrm{R}$, the buses in each of these components are either all included in $N$ or all excluded from $N$. (If $M$ is not a nodal cutset, then the network that remains when the buses in $M$ are removed from the network is connected and must be all of $N$, and area $\mathrm{R}$ must be the entire network. The theory works for this case, but it is less useful.)

The next step is to write the circuit equations for area $\mathrm{R}$, with the effect of the rest of the network replaced by the currents flowing into area $\mathrm{R}$ from the rest of the network. We use the following notation for column vectors of voltages and currents:

$V_{m} \quad$ voltages at border buses $M$;

$I_{m} \quad$ injected currents at border buses $M$;

$V_{n} \quad$ voltages at interior buses $N$;

$I_{n} \quad$ injected currents at interior buses $N$;

$V_{\bar{r}} \quad$ voltages at buses outside $\mathrm{R}$;

$I_{\bar{r}} \quad$ injected currents at buses outside $\mathrm{R}$.

The currents into border buses $M$ along lines not in $R$ are

$$
\begin{aligned}
I_{m}^{\text {into }} & =\sum_{j \in \bar{r}}\left(-G_{m j}\right)\left(V_{j}-V_{m}\right) \\
& =-G_{m \bar{r}} V_{\bar{r}}+\operatorname{diag}\left\{G_{m \bar{r}} \mathbf{1}\right\} V_{m}
\end{aligned}
$$

Here 1 is a row or a column vector of all ones. ${ }^{6}$ Order the buses so that all the border buses $M$ are first, all the interior buses $N$ are second, and all the buses outside $\mathrm{R}$ are third. Then Ohm's law $I=G V$ for the entire network is

$$
\left(\begin{array}{c}
I_{m} \\
I_{n} \\
I_{\bar{r}}
\end{array}\right)=\left(\begin{array}{ccc}
G_{m m} & G_{m n} & G_{m \bar{r}} \\
G_{n m} & G_{n n} & 0 \\
G_{\bar{r} m} & 0 & G_{\overline{r r}}
\end{array}\right)\left(\begin{array}{c}
V_{m} \\
V_{n} \\
V_{\bar{r}}
\end{array}\right)
$$

The first two block rows of (19) may be rewritten as

$$
\begin{aligned}
\left(\begin{array}{c}
I_{m} \\
I_{n}
\end{array}\right)= & \left(\begin{array}{cc}
G_{m m}^{\mathrm{R}} & G_{m n} \\
G_{n m} & G_{n n}
\end{array}\right)\left(\begin{array}{c}
V_{m} \\
V_{n}
\end{array}\right) \\
& -\left(\begin{array}{c}
I_{m}^{\text {into }} \\
0
\end{array}\right) \\
\text { where } \quad G_{m m}^{\mathrm{R}}= & G_{m m}+\operatorname{diag}\left\{G_{m \bar{r}} \mathbf{1}\right\} .
\end{aligned}
$$

The diagonal entries of $G_{m m}^{\mathrm{R}}$ are different than the submatrix $G_{m m}$ of the conductance matrix for the entire network, because

${ }^{6}$ The dimension of $\mathbf{1}$ varies with context and is chosen so that it can be multiplied by the matrix next to it in the formula. For example, given an $3 \times 4$ matrix $X, X 1=\sum_{j=1}^{4} X_{i j}$ is the column vector formed by adding the columns of $X$, and $1 X=\sum_{i=1}^{3} X_{i j}$ is the row vector formed by adding the rows of $X$. 


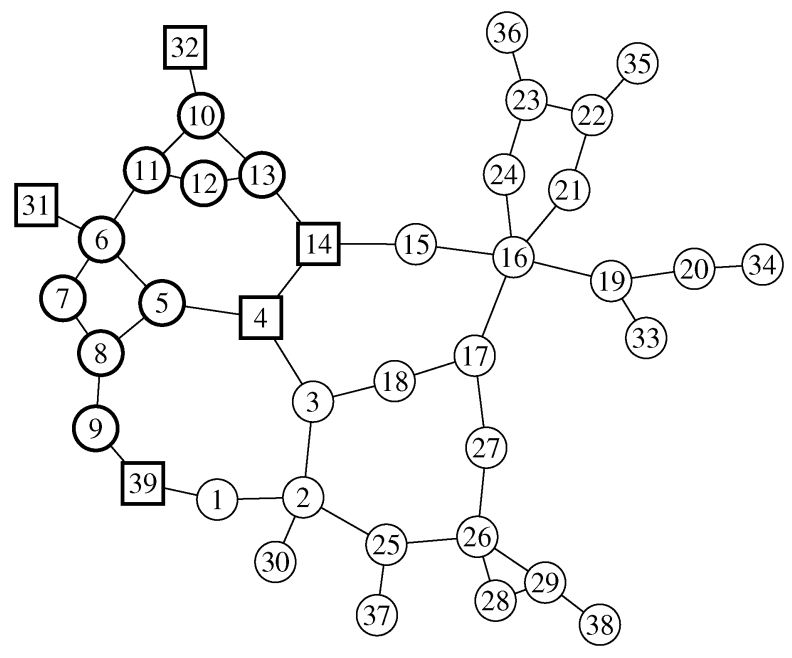

Fig. 2. Area R in the 39-bus New England test system. Interior buses $N=$ $\{5,6,7,8,9,10,11,12,13\}$ are shown with thick circles. Border buses $M=$ $\{31,32,39,4,14\}$ are shown with thick squares.
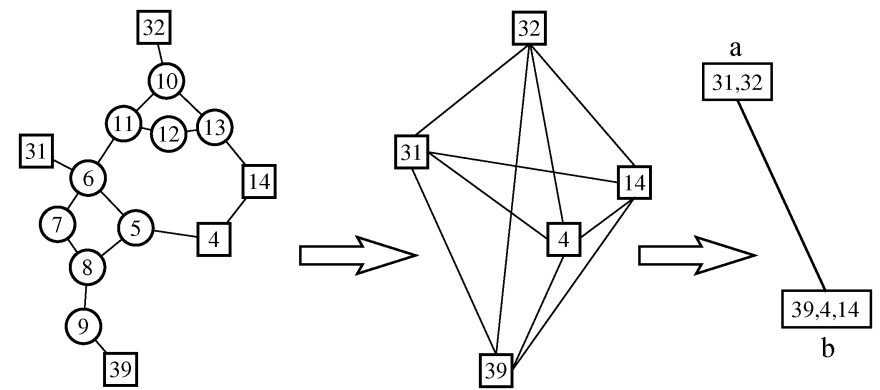

Fig. 3. Kron reduction of area $\mathrm{R}$ followed by contraction to two buses.

TABLE II

EXAMPLE RESUlTS FOR AREA R CONTRACTED TO Two BUSES

\begin{tabular}{c} 
DC load flow \\
$M_{a}=\{31,32\} \quad \hat{\theta}_{a b}=10.07 \quad P_{a b}=9.825 \quad b_{a b}=55.88$ \\
$M_{b}=\{39,4,14\}$ \\
$\hat{\theta}_{a b}=0.4587 \theta_{31}+0.5413 \theta_{32}-0.0863 \theta_{39}-0.4167 \theta_{4}-0.4970 \theta_{14}$ \\
\hline \multicolumn{3}{c}{ AC load flow } \\
$M_{a}=\{31,32\} \quad V_{a}=1.028 \angle 11.66 \quad V_{b}=0.9601 \angle 0.7634$ \\
$M_{b}=\{39,4,14\} \quad y_{a b}=1.614-56.57 j$ \\
$y_{a g}=0.0434+1.035 j \quad y_{b g}=-0.040+5.394 j$ \\
$V_{a}=(0.4858 \angle-0.40) V_{31}+(0.5143 \angle 0.38) V_{32}$ \\
$+(0.2058 \angle-0.01) V_{39}+(0.0687 \angle 2.11) V_{4}+(0.2744 \angle-179.5) V_{14}$ \\
$V_{b}=(0.0274 \angle-2.87) V_{31}+(0.0274 \angle 177.1) V_{32}$ \\
$+(0.2899 \angle-0.26) V_{39}+(0.4862 \angle-0.11) V_{4}+(0.2239 \angle 0.58) V_{14}$ \\
\hline
\end{tabular}

angles in degrees, all other quantities in per unit; $g$ is ground node

they do not include the conductances of the lines outside $\mathrm{R}$ that are joined to buses in $M$.

The currents entering the border buses of area $\mathrm{R}$ are the sum of the currents injected directly at the buses and the currents along the lines not in $\mathrm{R}$ that are joined to the buses:

$$
I_{m}^{\mathrm{R}}=I_{m}+I_{m}^{\text {into }} \text {. }
$$

Now (20) may be rewritten as

$$
\left(\begin{array}{c}
I_{m}^{\mathrm{R}} \\
I_{n}
\end{array}\right)=\left(\begin{array}{ll}
G_{m m}^{\mathrm{R}} & G_{m n} \\
G_{n m} & G_{n n}
\end{array}\right)\left(\begin{array}{c}
V_{m} \\
V_{n}
\end{array}\right)=G^{\mathrm{R}}\left(\begin{array}{c}
V_{m} \\
V_{n}
\end{array}\right)
$$

which is Ohm's law written for area R. $G^{\mathrm{R}}$ is the conductance matrix for the area $\mathrm{R}$ considered as a stand-alone network.

The next step reduces area $\mathrm{R}$ to an equivalent subnetwork. We retain the border buses $M$ and apply a standard Kron reduction ${ }^{7}$ to eliminate the interior buses $N$. Eliminating $V_{n}$ from (23) gives

$$
I_{m}^{\mathrm{R}}-G_{m n} G_{n n}^{-1} I_{n}=\left(G_{m m}^{\mathrm{R}}-G_{m n} G_{n n}^{-1} G_{n m}\right) V_{m}
$$

and, letting

$$
\begin{aligned}
I_{m}^{\mathrm{Rred}} & =I_{m}^{\mathrm{R}}-G_{m n} G_{n n}^{-1} I_{n} \\
G_{m m}^{\mathrm{Rred}} & =G_{m m}^{\mathrm{R}}-G_{m n} G_{n n}^{-1} G_{n m}
\end{aligned}
$$

we obtain a reduced subnetwork Rred electrically equivalent to $\mathrm{R}$ with the Ohm's law

$$
I_{m}^{\text {Rred }}=G_{m m}^{\text {Rred }} V_{m} .
$$

The reduced subnetwork Rred is the border buses $M$ joined by additional equivalent transmission lines and with additional current injections included in $I_{m}^{\text {Rred }}$ to account for the lines of the network outside $\mathrm{R}$ that inject currents into R.

The reduced subnetwork Rred consists of the border buses $M_{a}$ separated from the border buses $M_{b}$ by a cutset of lines exactly as considered in Section II. We now apply to Rred the formulas (14), (13), and (15) to define voltage $\hat{V}_{a b}$ across the area from $M_{a}$ to $M_{b}$, the area conductance $g_{a b}$, and the current $I_{a b}$ through the area from $M_{a}$ to $M_{b}$. That is, the reduced subnetwork Rred becomes a single line equivalent in which $M_{a}$ is contracted to a single bus $a$ and $M_{b}$ is contracted to a single bus $b$. The voltage across area $\mathrm{R}$ is the voltage $\hat{V}_{a b}$ between the buses of the single line equivalent, the conductance of area $\mathrm{R}$ is the conductance $g_{a b}$, and the current through area R is the current $I_{a b}$. The process of Kron reduction followed by contraction to the single line is illustrated in Fig. 3. Since both the Kron reduction and the contraction are consistent with circuit laws, the resulting area quantities satisfy Ohm's law (8).

An example of computational results for area $\mathrm{R}$ of the New England test system for DC and AC load flows is shown in Table II. (Parameters of the New England test system are specified in Appendix D.) The DC and AC load flows apply the resistive network results to different electrical quantities as shown in Table I. The voltage across area $\mathrm{R}$ is computed as the weighted combination of the voltages at the border buses shown in Table II. The weights correspond to (14). The voltage angle across area $\mathrm{R}$ is 10.1 degrees for the DC load flow and 10.9 degrees for the AC load flow. The AC load flow network includes shunts to a ground node denoted by $g$. Therefore, the single line equivalent also has shunts to the ground node. Accommodating the shunts and the ground node in the analysis is discussed in Appendix B.

\section{Generalizing Cutset Voltage to Voltages FOR SEVERAL SETS OF BUSES}

In Section II, we considered a connected resistive network in which the buses were partitioned into two subsets $M_{a}$ and $M_{b}$. The cutset voltage defined a voltage between two subsets

${ }^{7}$ According to $[5$, Theorem $(5,7)]$ and $[6$, Lemma 2.1], for a real conductance matrix $G^{\mathrm{R}}$, area R connected implies that $G^{\mathrm{R}}$ has rank one less than the number of buses in R, $G_{n n}$ is not singular, and the Kron reduction is valid. We thank F. Dörfler for providing these references. Kron reduction is also known as a Ward equivalent or a Schur reduction. 


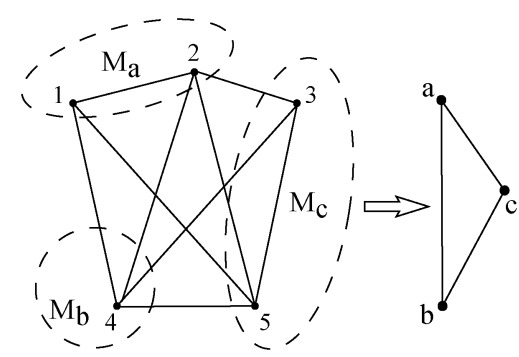

Fig. 4. Contraction of a network to three buses.

of buses $M_{a}$ and $M_{b}$. As illustrated in Fig. 1, the network was contracted to two buses joined by a single line equivalent.

Now we generalize by partitioning the network into three nonempty subsets $M_{a}, M_{b}, M_{c}$ and obtaining the voltages of these three subsets of buses and the conductances between them. This contracts the network to three buses joined by three equivalent lines. The $a$ bus of the reduced network corresponds to the buses $M_{a}$ of the original network, the $b$ bus corresponds to $M_{b}$, and the $c$ bus corresponds to $M_{c}$ as illustrated in Fig. 4. We want to calculate the voltages $V_{a}, V_{b}, V_{c}$ of the three buses, the conductances $g_{a b}, g_{b c}, g_{c a}$ of the three equivalent lines, and the equivalent currents $I_{a}, I_{b}, I_{c}$ injected at the three buses.

We write

$$
V_{A}=\left(\begin{array}{c}
V_{a} \\
V_{b} \\
V_{c}
\end{array}\right) \text { and } I_{A}=\left(\begin{array}{c}
I_{a} \\
I_{b} \\
I_{c}
\end{array}\right) .
$$

The conductance matrix of the equivalent 3 bus network is

$$
G_{A}=\left(\begin{array}{ccc}
g_{a b}+g_{c a} & -g_{a b} & -g_{c a} \\
-g_{a b} & g_{a b}+g_{b c} & -g_{b c} \\
-g_{c a} & -g_{b c} & g_{b c}+g_{c a}
\end{array}\right) .
$$

The matrix $\sigma_{A}$ defines the buses in each of $M_{a}, M_{b}, M_{c}$ by

$$
\left(\sigma_{A}\right)_{i j}= \begin{cases}1 & \text { bus } j \text { in } M_{a} \text { and } i=1 \\ 1 & \text { bus } j \text { in } M_{b} \text { and } i=2 \\ 1 & \text { bus } j \text { in } M_{c} \text { and } i=3 \\ 0 & \text { otherwise. }\end{cases}
$$

For example, the first row of $\sigma_{A}$ defines the buses in $M_{a}$ and corresponds to the row vector $\sigma_{a}$ in (10). As in Section II-B, the bus voltages are $V_{m}$, the bus current injections are $I_{m}^{\text {red }}$, the network conductance matrix is $G_{m m}^{\text {red }}$, and the Ohm's law equations for the network are

$$
I_{m}^{\mathrm{red}}=G_{m m}^{\mathrm{red}} V_{m} .
$$

The injected currents $I_{a}$ at bus $a$ of the three-bus network must be the sum of the currents injected at the buses in $M_{a}$. Similarly, $I_{b}$ is the sum of the currents injected at the buses in $M_{b}$ and $I_{c}$ is the sum of the currents injected at the buses in $M_{c}$. This can be written

$$
I_{A}=\sigma_{A} I_{m}^{\mathrm{red}} .
$$

We obtain the conductance matrix of the three bus network as

$$
G_{A}=\sigma_{A} G_{m m}^{\mathrm{red}} \sigma_{A}^{T} \text {. }
$$

It can be seen that (32) generalizes (13). It follows from (32) and $G_{m m}^{\text {red }}$ irreducible that $G_{A}$ is irreducible. Moreover, $G_{A}$ has rank two. ${ }^{7}$
The voltage $V_{A}$ is defined to be a solution to

$$
G_{A} V_{A}=\sigma_{A} G_{m m}^{\mathrm{red}} V_{m} .
$$

Allowing for the different position in the equation of the conductance matrix $G_{A}$, it can be seen that (33) generalizes (14). It follows from (33) that multiplying (30) by $\sigma_{A}$ yields

$$
I_{A}=\sigma_{A} I_{m}^{\mathrm{red}}=\sigma_{A} G_{m m}^{\mathrm{red}} V_{m}=G_{A} V_{A}
$$

which is Ohm's law for the three-bus network.

Now we discuss solving (33) for $V_{A}$. Although $G_{A}$ is singular, (33) can be solved in a standard way. First note that since $1 \sigma_{A}=1$ and the rows of $G_{m m}^{\text {red }}$ sum to zero

$$
1 \sigma_{A} G_{m m}^{\mathrm{red}}=1 G_{m m}^{\mathrm{red}}=0 .
$$

Then the components of the right-hand side of (33) sum to zero and are in the range of $G_{A}$. Moreover, since $G_{A}$ has rank two, (33) is solvable for $V_{A}$ to within a constant added to each component of $V_{A}$. The constant is determined by the choice of zero reference voltage. For example, we can require the solution $V_{A}$ to have its third component $V_{c}=0$. Then we can calculate the first two components $V_{a}$ and $V_{b}$ by solving the equations formed by deleting the last row of (33).

The calculation of $V_{A}, I_{A}$, and $G_{A}$ is given here for the case in which the buses are partitioned into three subsets $M_{a}, M_{b}$, and $M_{c}$, but it is clear that the corresponding calculation for two subsets or for more than three subsets is the same except for the dimensions of the vectors and matrices. We now calculate $V_{A}$ in the case of two subsets $M_{a}$ and $M_{b}$ in order to make it clear that it gives the same voltage difference $\hat{V}_{a b}=V_{a}-V_{b}$ that is the cutset voltage calculated in Section II-B.

Suppose that the network buses are partitioned into two subsets $M_{a}, M_{b}$. The conductance matrix of the equivalent single line and two bus network is

$$
G_{A}=\left(\begin{array}{cc}
g_{a b} & -g_{a b} \\
-g_{a b} & g_{a b}
\end{array}\right) .
$$

The rows of the matrix $\sigma_{A}$ are the row vectors $\sigma_{a}$ and $\sigma_{b}$ :

$$
\sigma_{A}=\left(\frac{\sigma_{a}}{\sigma_{b}}\right) \text {. }
$$

First we note that

$$
I_{A}=\left(\begin{array}{c}
I_{a} \\
I_{b}
\end{array}\right)=\sigma_{A} I_{m}^{\mathrm{red}}=\left(\begin{array}{c}
\sigma_{a} \\
\sigma_{b}
\end{array}\right) I_{m}^{\mathrm{red}}=\left(\begin{array}{c}
I_{a b} \\
I_{b a}
\end{array}\right)
$$

agrees with the definition of $I_{a b}$ in (8). Now we consider the other quantities. The conductance matrix of the single line equivalent is

$$
G_{A}=\sigma_{A} G_{m m}^{\mathrm{red}} \sigma_{A}^{T}=\left(\begin{array}{cc}
\sigma_{a} G_{m m}^{\mathrm{red}} \sigma_{a}^{T} & \sigma_{a} G_{m m}^{\mathrm{red}} \sigma_{b}^{T} \\
\sigma_{b} G_{m m}^{\mathrm{red}} \sigma_{a}^{T} & \sigma_{b} G_{m m}^{\mathrm{red}} \sigma_{b}^{T}
\end{array}\right) .
$$

Since $\sigma_{b}=1-\sigma_{a}$

$G_{A}=\left(\begin{array}{cc}\sigma_{a} G_{m m}^{\mathrm{red}} \sigma_{a}^{T} & -\sigma_{a} G_{m m}^{\mathrm{red}} \sigma_{a}^{T} \\ -\sigma_{a} G_{m m}^{\mathrm{red}} \sigma_{a}^{T} & \sigma_{a} G_{m m}^{\mathrm{red}} \sigma_{a}^{T}\end{array}\right)=\left(\begin{array}{cc}g_{a b} & -g_{a b} \\ -g_{a b} & g_{a b}\end{array}\right)$.

Equation (33) becomes

$$
\begin{aligned}
\left(\begin{array}{cc}
g_{a b} & -g_{a b} \\
-g_{a b} & g_{a b}
\end{array}\right)\left(\begin{array}{c}
V_{a} \\
V_{b}
\end{array}\right) & =\left(\begin{array}{c}
\sigma_{a} G_{m m}^{\mathrm{red}} \\
\sigma_{b} G_{m m}^{\mathrm{red}}
\end{array}\right) V_{m} \\
& =\left(\begin{array}{c}
\sigma_{a} G_{m m}^{\mathrm{red}} \\
-\sigma_{a} G_{m m}^{\mathrm{red}}
\end{array}\right) V_{m}
\end{aligned}
$$



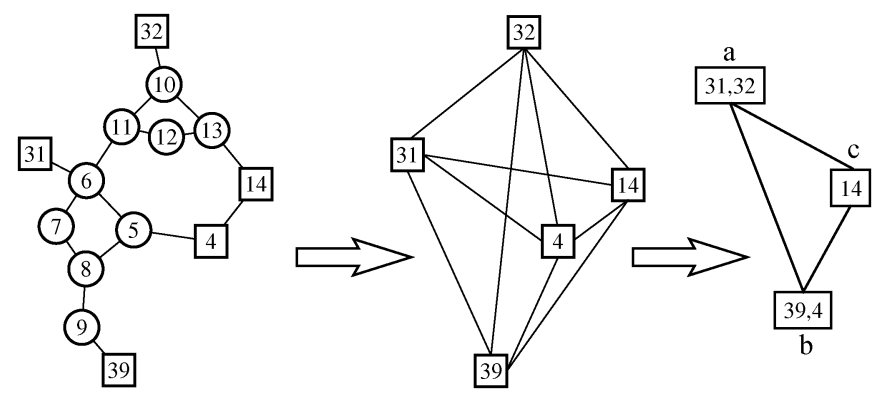

Fig. 5. Kron reduction of area $\mathrm{R}$ followed by contraction to three buses.

TABLE III

EXAmple Results for AREa R Contracted to Three Buses

\begin{tabular}{lll}
\hline \multicolumn{3}{c}{ DC load flow } \\
$M_{a}=\{31,32\}$ & $\theta_{a}=9.141 \quad P_{a}=9.825$ \\
$M_{b}=\{39,4\}$ & $\theta_{b}=-1.855$ & $P_{b}=-8.344$ \\
$M_{c}=\{14\}$ & $\theta_{c}=0 \quad$ & $P_{c}=-1.482$ \\
\multicolumn{2}{c}{$b_{a b}=28.11 \quad b_{b c}=91.06 \quad b_{c a}=27.77$} \\
$\theta_{a}=0.4308 \theta_{31}+0.5692 \theta_{32}-0.0637 \theta_{39}+0.0637 \theta_{4}-\theta_{14}$ \\
$\theta_{b}=-0.0554 \theta_{31}+0.0554 \theta_{32}+0.0450 \theta_{39}+0.9551 \theta_{4}-\theta_{14}$
\end{tabular}

angles in degrees, all other quantities in per unit

and multiplying both sides by the row vector $(1 / 2,-1 / 2)$ yields

$$
g_{a b}\left(V_{a}-V_{b}\right)=\sigma_{a} G_{m m}^{\mathrm{red}} V_{m} .
$$

Hence, comparing (39) with (14), $\hat{V}_{a b}=V_{a}-V_{b}$.

\section{Generalizing Voltage Across an Area to Multiple Voltages Across an AREA}

Given the generalization of the cutset voltage to voltages for several sets of buses in Section IV, it is straightforward to generalize to multiple voltages across an area. We consider the case of three voltages. Following the pattern of Section III, choose a connected area $\mathrm{R}$ with border buses $M$. Partition the border buses into subsets of buses $M_{a}, M_{b}, M_{c}$. As in Section III, form the conductance matrix $G^{\mathrm{R}}$ of the area $\mathrm{R}$ and apply Kron reduction to eliminate the interior buses. The result is the equivalent network Rred with border buses $M=M_{a} \bigcup M_{b} \bigcup M_{c}$ joined by equivalent lines with bus voltages $V_{m}$, equivalent bus current injections $I_{m}^{\text {red }}$, and equivalent conductance matrix $G_{m m}^{\mathrm{Rred}}$. $G_{m m}^{\mathrm{Rred}}$ is guaranteed to inherit the symmetric and irreducible properties of $G^{\mathrm{R}}$ [6, Lemma 2.1]. Now apply the calculations of Section IV to this Kron-reduced subnetwork to obtain voltages $V_{a}, V_{b}, V_{c}$, line conductances $g_{a b}, g_{b c}, g_{c a}$, and current injections $I_{a}, I_{b}, I_{c}$ for area $\mathrm{R}$.

For example, in Fig. 5, the Kron reduction of area $\mathrm{R}$ is contracted to three buses. A DC load flow example computation of the voltage angles, power injections, and susceptances of the three-bus network is shown in Table III.

\section{Simultaneous Reduction of Multiple AREas}

The previous sections have analyzed a single area. In model reduction, or for monitoring multiple areas, it can be convenient to obtain a reduced model of the entire network by reducing multiple areas simultaneously. This section discusses the simultaneous reduction for the three areas $R_{\gamma}, R_{\alpha}, R_{\beta}$ shown in

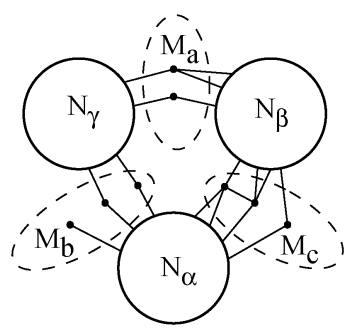

Fig. 6. Areas $R_{\gamma}=N_{\gamma} \cup M_{a} \cup M_{b}, R_{\alpha}=N_{\alpha} \cup M_{b} \cup M_{c}$, and $R_{\beta}=$ $N_{\beta} \cup M_{c} \cup M_{a}$.

Fig. 6. Write $M=M_{a} \cup M_{b} \cup M_{c}$ for the set of all the border buses of all three areas and $N=N_{\gamma} \cup N_{\alpha} \cup N_{\beta}$ for all the interior buses. Each border bus is either on the border of two contiguous areas or on the border of only one area.

Order the buses so that all the border buses $M$ come first. Then Ohm's law for the entire network is

$$
\left(\begin{array}{c}
I_{m} \\
I_{n}
\end{array}\right)=\left(\begin{array}{ll}
G_{m m} & G_{m n} \\
G_{n m} & G_{n n}
\end{array}\right)\left(\begin{array}{c}
V_{m} \\
V_{n}
\end{array}\right)
$$

The next step is Kron reduction to an equivalent network that retains only the border buses $M$. The equivalent current injections and equivalent conductance matrix of the Kron reduced network are

$$
\begin{aligned}
I_{m}^{\mathrm{red}} & =I_{m}-G_{m n} G_{n n}^{-1} I_{n} \\
G_{m m}^{\mathrm{red}} & =G_{m m}-G_{m n} G_{n n}^{-1} G_{n m} .
\end{aligned}
$$

The rows of the matrix $\sigma_{A}$ define the buses in $M_{a}, M_{b}, M_{c}$ according to

$$
\left(\sigma_{A}\right)_{i j}= \begin{cases}1 & i=1 \text { and bus } j \text { in } M_{a} \\ 1 & i=2 \text { and bus } j \text { in } M_{b} \\ 1 & i=3 \text { and bus } j \text { in } M_{c} \\ 0 & \text { otherwise. }\end{cases}
$$

For the voltages and injected currents injected at $M_{a}, M_{b}, M_{c}$, we write

$$
V_{A}=\left(\begin{array}{c}
V_{a} \\
V_{b} \\
V_{c}
\end{array}\right) \quad \text { and } \quad I_{A}=\left(\begin{array}{c}
I_{a} \\
I_{b} \\
I_{c}
\end{array}\right) .
$$

Then, with this slightly revised notation, the formulas for the voltages $V_{A}$ at $M_{a}, M_{b}, M_{c}$, the conductance matrix $G_{A}$ between $M_{a}, M_{b}, M_{c}$, and the injected currents $I_{A}$ at $M_{a}, M_{b}, M_{c}$, are (33), (32), (31). Moreover, the Ohm's law (34) applies. Appendix C shows that this simultaneous reduction of the three areas gives the same results as considering each area separately.

For example, as applied to DC load flow and four areas, consider Fig. 7. In Fig. 7, the entire New England 39-bus system is contracted to an equivalent network with five buses. An example computation of the voltage angles, power injections, and susceptances of the five-bus network is shown in Table IV.

\section{MONITORING AREA STRESS}

This section shows an application of area angles to monitor area stress from synchrophasor measurements around the border of the area. 


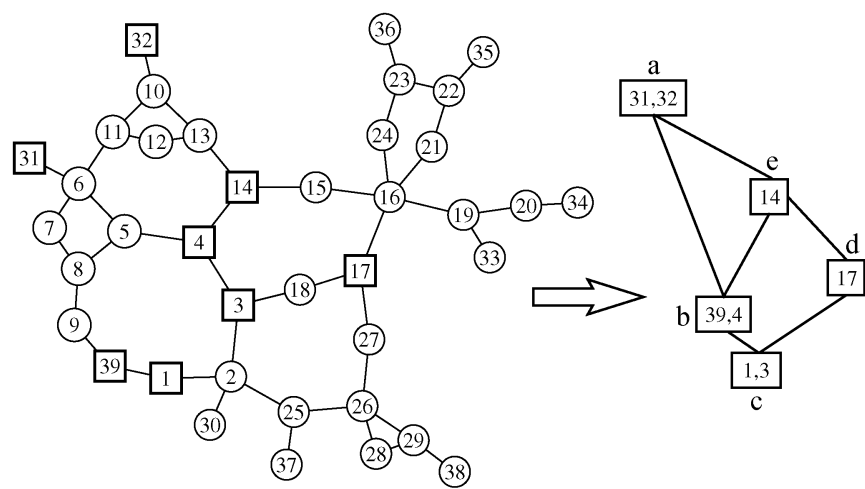

Fig. 7. New England 39-bus test system contracted to five buses. Border buses separating areas are shown with squares.

TABLE IV

EXAMPLE RESUlTS For ENTIRE NETWORK CONTRACTED to FiVE Buses

\begin{tabular}{|c|c|c|c|c|}
\hline \multicolumn{5}{|c|}{ DC load flow } \\
\hline$M_{a}=\{31,32\}$ & $\theta_{a}=\varsigma$ & .115 & $P_{a}$ & $=9.825$ \\
\hline$M_{b}=\{39,4\}$ & $\theta_{b}=-$ & -1.906 & $P_{b}$ & $=-10.17$ \\
\hline$M_{c}=\{1,3\}$ & $\theta_{c}=-$ & -0.7617 & $P_{c}$ & $=0.7745$ \\
\hline$M_{d}=\{17\}$ & $\theta_{d}=c$ & .1823 & $P_{d}$ & $=1.042$ \\
\hline$M_{e}=\{14\}$ & $\theta_{e}=0$ & & $P_{e}$ & $=-1.468$ \\
\hline $\begin{array}{ll}b_{a b}=28.11 & b_{a c} \\
& b_{b c}\end{array}$ & $\begin{array}{l}0 \\
86.95\end{array}$ & $\begin{aligned} b_{a d} & =0 \\
b_{b d} & =0 \\
b_{c d} & =58\end{aligned}$ & & $\begin{aligned} b_{a e} & =27.77 \\
b_{b e} & =91.06 \\
b_{c e} & =0 \\
b_{d e} & =25.00\end{aligned}$ \\
\hline
\end{tabular}

angles in degrees, all other quantities in per unit

\section{A. Background}

Synchronized phasor measurements are being widely deployed for power system monitoring and control [7]. Here we assume the availability of suitable synchrophasor measurements at a control center. Tate and Overbye [8] give a good discussion of the necessary signal processing.

Previous work on monitoring power system stress with phasor measurements has focused on the angle difference between two buses. Simulations of the grid conditions before the August 2003 USA/Canada blackout show increasing angle differences between Cleveland and West Michigan, suggesting that large angle differences could be a blackout precursor [9]. Simulations of the New England grid show that angle differences are the best phasor measurements in discriminating alert and emergency states [10].

A large angle difference between two buses does indicate, in a general sense, a stressed power system. However, this angle difference is affected by changes throughout the entire grid, and it is difficult to interpret changes in the angle difference or set thresholds.

It is natural to measure the stress on an individual transmission line by the angle difference across the line. Since the preceding sections show how an area can be reduced to an equivalent transmission line, and the angle across the area is the angle across the equivalent transmission line, we can use the angle across the area as a measure of the area stress.

For further motivation, consider the DC load flow of two buses $a$ and $b$ joined by two equal lines. We measure the stress between $a$ and $b$ by the phasor angle difference $\theta_{a}-\theta_{b}$. In this simple case, the reduction to a single line is clear: $\hat{\theta}_{a b}=$ $\theta_{a}-\theta_{b}, b_{a b}$ is the sum of the line susceptances, and $P_{a b}$ is the sum of the line power flows. $\hat{\theta}_{a b}$ measures the stress better than $P_{a b}$, because if one of the lines outages, then the power flow $P_{a b}$ (now flowing only on the line remaining in service) does not change but $\hat{\theta}_{a b}$ doubles, correctly indicating the increased stress.

\section{B. Stress Across an Area}

We use a DC load flow model of an area $\mathrm{R}$ with border buses $M_{a}$ and $M_{b}$. Then the area angle $\hat{\theta}_{a b}$ gives the stress across area R from $M_{a}$ to $M_{b}$. The Ohm's law $P_{a b}=b_{a b} \hat{\theta}_{a b}$ corresponding to (8) implies that $\hat{\theta}_{a b}$ is proportional to the real power $P_{a b}$ passing through the area and inversely proportional to the area susceptance $b_{a b}$.

By using Table I to substitute the corresponding DC load flow circuit quantities for the resistive circuit quantities in (14), the area angle $\hat{\theta}_{a b}$ is a weighted linear combination of the border bus angles $\theta_{m}$ :

$$
\hat{\theta}_{a b}=w_{m} \theta_{m}, \text { where } w_{m}=\frac{\sigma_{a} B_{m m}^{\mathrm{red}}}{b_{a b}} .
$$

The weights in the row vector $w_{m}$ are determined from the DC load flow model of R via (45), (12), and (13). Therefore, if we place phasor angle measurements at all the border buses of $\mathrm{R}$ and know the status of lines in $\mathrm{R}$, we can easily determine the angle $\hat{\theta}_{a b} .{ }^{8}$ The availability in practice of a DC load flow model of an area is discussed in [8].

We illustrate stress across the area R shown in Fig. 8, which shows part of a 225-bus model of the Western North American power grid. ${ }^{9}$ The three border buses with assumed phasor measurements are $M_{a}=\{$ ELDORADO, PALOVRDE $\}$ and $M_{b}=\{$ VINCENT $\}$. From (45), the angle $\hat{\theta}_{a b}$ east to west across area $\mathrm{R}$ combines the border buses angles as

$\hat{\theta}_{a b}=0.7987 \theta_{\text {ELDORADO }}+0.2013 \theta_{\mathrm{PALOVRDE}}-\theta_{\mathrm{VINCENT}}$.

The base case area stress is $\hat{\theta}_{a b}=10.85$ degree and the equivalent power flow westward through the area is $P_{a b}=1734 \mathrm{MW}$. The area susceptance is $b_{a b}=91.62$ per unit on a $100 \mathrm{MW}$ base.

Examples of transfers and line outages are shown in Fig. 8. Table $\mathrm{V}$ shows the effect of these transfers and line outages on the angle $\hat{\theta}_{a b}$ across area $\mathrm{R}$. The transfer inside area $\mathrm{R}$ increases $\hat{\theta}_{a b}$ by increasing the effective westward power flow. The transfer outside area $\mathrm{R}$ also increases the westward power flow through the area and $\hat{\theta}_{a b}$. The line outages also increase $\hat{\theta}_{a b}$. In particular, the line outage inside $\mathrm{R}$ decreases the area admittance whereas the line outage outside $\mathrm{R}$ has the effect of increasing the westward power transfer through the area.

\section{Internal and External Stress Angles}

Section VII-B shows that the area angle $\hat{\theta}_{a b}$ measures the total east-west stress across area $\mathrm{R}$, and it responds to changes both inside and outside the area. Now we decompose the area angle into an internal angle due to power injections inside the

\footnotetext{
${ }^{8}$ Alternatively, the AC load flow version of (45) may be obtained by substituting the complex circuit quantities according to Table I. This yields an expression for the complex voltage across the area in terms of the complex voltages measured at the border buses.

${ }^{9}$ We thank C.-C. Liu, N. Yu, and J. Li for graciously providing this power system model.
} 


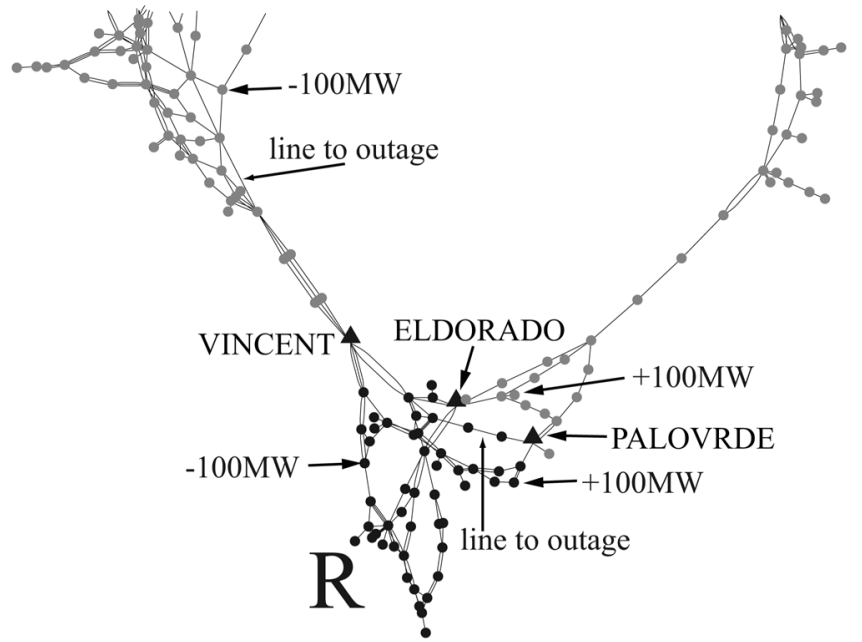

Fig. 8. Area R shown as the black buses; all other buses are gray. The border buses with phasor measurements are the labelled black triangles. A $100 \mathrm{MW}$ power transfer and a line to outage are shown both inside $\mathrm{R}$ (in the black buses) and outside $\mathrm{R}$ (in the gray buses).

TABLE V

AREA ANGLES

\begin{tabular}{cccc} 
& $\hat{\theta}_{a b}$ & $\hat{\theta}_{a b}^{\text {into }}$ & $\hat{\theta}_{a b}^{\text {inR }}$ \\
\hline base case & 10.85 & 9.776 & 1.070 \\
transfer 100 MW inside R & 11.10 & 9.765 & 1.330 \\
transfer 100 MW outside R & 11.45 & 10.38 & 1.070 \\
line outage inside R & 13.36 & 9.660 & 3.702 \\
line outage outside R & 11.23 & 10.16 & 1.070 \\
\hline \multicolumn{4}{c}{ angles in degrees }
\end{tabular}

area and an external angle due to power flows from other areas. The internal and external area angles offer more specific stress information about the area.

Based on (15), (24), and (22), the power flowing from $M_{a}$ to $M_{b}$ decomposes as

$$
P_{a b}=P_{a}^{\text {into }}+P_{a}^{\mathrm{inR}}
$$

$P_{a}^{\text {into }}$ is the power flowing into area $\mathrm{R}$ along the external tie lines attached to the border buses in $M_{a}$. $P_{a}^{\text {inR }}=\sigma_{a} P_{m}-$ $\sigma_{a} B_{m n} B_{n n}^{-1} P_{n}$ is the total equivalent power injected into the buses $M_{a}$ that corresponds to power injected in R. Moreover, since $P_{a b}=-P_{b a}$

$$
\begin{aligned}
P_{a b} & =P_{a}^{\text {into }}+P_{a}^{\mathrm{inR}}=-P_{b}^{\mathrm{into}}-P_{b}^{\mathrm{inR}} \\
& =\frac{1}{2}\left(P_{a}^{\mathrm{into}}-P_{b}^{\mathrm{into}}\right)+\frac{1}{2}\left(P_{a}^{\mathrm{inR}}-P_{b}^{\mathrm{inR}}\right) .
\end{aligned}
$$

Equation (47) decomposes $P_{a b}$ into two parts. The first part is related to the difference of the external power flows injected at buses $M_{a}$ and $M_{b}$. The second part is related to the difference of the power flows equivalent to the power injected in area $\mathrm{R}$. Dividing (47) by the susceptance $b_{a b}$ and using the Ohm's law (8) decomposes the angle across the area as

$$
\begin{aligned}
\hat{\theta}_{a b} & =\hat{\theta}_{a b}^{\text {into }}+\hat{\theta}_{a b}^{\text {inR }}, \quad \text { where } \\
\hat{\theta}_{a b}^{\text {into }} & =\frac{P_{a}^{\text {into }}-P_{b}^{\text {into }}}{2 b_{a b}} \text { and } \quad \hat{\theta}_{a b}^{\text {inR }}=\frac{P_{a}^{\text {inR }}-P_{b}^{\text {inR }}}{2 b_{a b}} .
\end{aligned}
$$

The angle $\hat{\theta}_{a b}^{\text {into }}$ is caused by differences in the powers entering into the area at $M_{a}$ and $M_{b}$ and measures the external stress on area R. The angle $\hat{\theta}_{a b}^{\mathrm{inR}}$ is caused by the differences at $M_{a}$ and $M_{b}$ of the powers equivalent to the powers generated or consumed inside area $\mathrm{R}$ and measures the internal stress on $\mathrm{R}$. In particular, $\hat{\theta}_{a b}^{\mathrm{inR}}$ only depends on the generation and loads inside $\mathrm{R}$ and the lines in service inside $\mathrm{R}$.

The internal angle $\hat{\theta}_{a b}^{\text {inR }}$ can be obtained from additional tie line phasor measurements as follows. The tie line power flowing into a bus can be obtained from phasor measurements if both the currents in the tie lines and the bus voltage are measured. If the external tie line power entering each bus in $M_{a}$ is known from these measurements, these can be summed to obtain the total power $P_{a}^{\text {into }}$ entering into the area R through $M_{a}$. The total power $P_{b}^{\text {into }}$ entering into R through $M_{b}$ can be obtained similarly. Then we can obtain $\hat{\theta}_{a b}^{\text {into }}$ using (49) and then obtain $\hat{\theta}_{a b}^{\text {inR }}$ using $\hat{\theta}_{a b}^{\text {inR }}=\hat{\theta}_{a b}-\hat{\theta}_{a b}^{\text {into }}$.

Line outages can be modeled by equal and opposite power injections at either end of the line whose magnitude is proportional to the power flow on the line before it outaged [8]. Since the angles across the area respond proportionally to the magnitude of the equal and opposite power injections, the change in the area angles when the line outages is proportional to the power flow on the line before it outaged. Formulas quantifying this relationship using generalized line outage distribution factors are given in [4].

In the example of area $\mathrm{R}$ in Fig. 8, the base case area angle $\hat{\theta}_{a b}=10.85$ degree decomposes into the external angle $\hat{\theta}_{a b}^{\text {into }}=$ 9.776 degree and the internal angle $\hat{\theta}_{a b}^{\mathrm{inR}}=1.070$ degree. It can be seen from Table $\mathrm{V}$ that all the transfers and line outages shown in Fig. 8 affect the external angle $\hat{\theta}_{a b}^{\text {into }}$. However, the internal angle $\hat{\theta}_{a b}^{\mathrm{inR}}$ only changes when the transfer or line outage is inside area $\mathrm{R}$.

\section{Discussion}

We suggest using total, internal, and external area angles to monitor stress across a power system area. The angles can be easily calculated from phasor measurements at all the buses along the border of the area using a DC load flow model of the area and knowledge of which lines in the area are in service. It is natural to place phasor measurements at area tie lines. The stress monitoring shows one way to gain value from placing phasor measurements at all the tie lines to an area.

Previous approaches to measuring stress with phasor measurements have used the difference of angles at two buses or searched for patterns in angles from many buses. The area angles have some advantages over these approaches. The area angles give stress information specific to an area of the power system. This corresponds with the way large power systems are operated, and information that describes specific properties of a specific area of a large power system is more actionable. The area angles respond to line outages and power redispatches in accordance with circuit laws. The internal angle only changes when lines inside the area outage or when there is redispatch of power within the area, and can therefore be used to detect changes and confirm that they happened inside the area.

\section{CONCLUSION}

This paper defines and explains from first principles a new concept of voltages across an area of a network. The new volt- 
ages are weighted combinations of voltages at the border buses of the area. The voltages can be real voltages in a resistive network, voltage angles in a DC load flow, or complex voltages in an AC load flow. Ohm's law is satisfied in each of these cases as detailed in Table I. The voltages across an area are obtained by a standard Kron reduction of the area to a network joining the border buses, followed by a contraction of the border buses into a network equivalent to the area that typically has two or three buses. The contraction of the border buses relies on a new concept of voltage across a cutset of lines.

Voltage angles across an area are a combination of individual bus voltages at the border of the area. The area conductance and currents through the area also combine together individual line conductances and individual currents to describe bulk electrical characteristics of the area. (Remember to substitute these quantities appropriately for DC and AC load flow as shown in Table I; for example, in DC load flow, the "currents" become real powers.) Since the area voltages, conductances, and currents obey circuit laws, they are more meaningful and useful than an arbitrary combination of circuit quantities. In particular, they will behave in ways consistent with the intuition of power system operators and engineers.

The applications of the new area voltages include model reduction, monitoring area stress, and detecting line trips from synchrophasor measurements [2]-[4]. The explanations of area voltages in this paper illustrate systematic AC and DC load flow reductions for single and multiple areas. We also describe how area angles measure the area stress and can be obtained from synchrophasor measurements of voltages at the border buses of an area. If the currents or powers flowing into the area at the border buses are also measured, then an internal angle that only responds to changes inside the area can also be monitored. We refer to [4] for initial work applying area angles to detecting line trips in areas.

All of these applications depend on a systematic circuit theory foundation and formulas to compute the area voltages, conductances, and currents. This paper provides this foundation and derives those formulas.

\section{APPENDIX A \\ Cutset Voltage Derived From Network Theory}

We derive the cutset voltage $\hat{V}_{a b}$ as an instance of classical circuit theory using a novel and nonstandard choice of basis. Chen in [1] explains a generalized cutset analysis of a resistive network with voltage and current sources. We now state Chen's equations. Let the rows of the matrix $Q$ specify a basis for the cutset vector space of the network. (The cutset vector space of the network is spanned by the rows of $A$ and has dimension nbus -1 for a connected network [1], [11].) Write $\hat{V}_{q}$ for the (generalized) cutset voltages, $I_{q}$ for the (generalized) cutset currents, $G_{q}$ for the (generalized) cutset admittance matrix, and $I_{b}$ for the branch current flows. Then

$$
\begin{aligned}
\hat{V} & =Q^{T} \hat{V}_{q} \\
I_{q} & =Q I_{b} \\
G_{q} & =Q \Lambda Q^{T} \\
\text { and } \quad I_{q} & =G_{q} \hat{V}_{q}
\end{aligned}
$$

which is Chen's equation (2.81). Another good explanation of the cutset vector space and (50)-(53) is by Bryant in [11, section 8], where (53) appears as Bryant's equation (56).
Now we make a special choice of the basis for the cutset vector space of the network by choosing the first row of the $Q$ matrix to be the cutset $c$ so that

$$
Q=\left(\begin{array}{c}
c \\
Q_{\perp}
\end{array}\right)
$$

and so that the remaining rows $Q_{\perp}$ of $Q$ are basis vectors of the cutset vector space orthogonal to $c$ in the sense that

$$
c \Lambda Q_{\perp}^{T}=0 .
$$

(It is often impossible to choose all rows of $Q_{\perp}$ to consist of vectors with entries \pm 1 and zero corresponding to the usual cutsets, but this causes no fundamental difficulty.) Then in the basis (54), multiplying (50) on the left with $c \Lambda$ gives

$$
c \Lambda \hat{V}=c \Lambda Q^{T} \hat{V}_{q}=c \Lambda c^{T} \hat{V}_{q 1}=g_{a b} \hat{V}_{q 1}
$$

where $\hat{V}_{q 1}$, the first component of $\hat{V}_{q}$, is the generalized cutset coordinate associated with $c$. Comparison of (56) with (7) shows that $\hat{V}_{a b}=\hat{V}_{q 1}$ is the generalized cutset coordinate associated with $c$ in the basis (54). Moreover, in the basis (54), we have from (52) that

$$
G_{q}=Q \Lambda Q^{T}=\left(\begin{array}{cc}
c \Lambda c^{T} & 0 \\
0 & Q_{\perp} \Lambda Q_{\perp}^{T}
\end{array}\right)=\left(\begin{array}{cc}
g_{a b} & 0 \\
0 & Q_{\perp} \Lambda Q_{\perp}^{T}
\end{array}\right)
$$

and the first components of (51) and (53) become, respectively,

$$
I_{q 1}=c I_{b}=\sigma_{a} A I_{b}=\sigma_{a} I_{m}^{\mathrm{red}}=I_{a b} \quad \text { and } \quad I_{q 1}=g_{a b} \hat{V}_{q 1} .
$$

Hence, the first component of (53) may be written as $I_{a b}=$ $g_{a b} \hat{V}_{a b}$, which is (17). Thus, we have found a nonstandard cutset basis (54) in which the cutset voltage $\hat{V}_{a b}$ is the generalized cutset coordinate associated with the basis element $c$.

As an example, consider the network of Fig. 1 with all the line conductances equal to 1 . Then $\Lambda$ is an identity matrix and a $Q$ matrix satisfying (55) is

$$
Q=\left(\begin{array}{ccccccccc}
1 & 1 & 1 & 1 & 1 & 1 & 0 & 0 & 0 \\
1 & 3 & -2 & 0 & -2 & 0 & 3 & 0 & 2 \\
-2 & 0 & 1 & 3 & -2 & 0 & -3 & 3 & 2 \\
-2 & 0 & -2 & 0 & 1 & 3 & 0 & -3 & 2
\end{array}\right) .
$$

\section{APPENDIX B \\ ShunTs TO A GROUND NODE}

Sometimes the area $\mathrm{R}$ has all buses joined by shunts to a ground node $g$ that is only implicitly included in R. This appendix reviews the adjustments needed.

Ohm's law for area $\mathrm{R}$ together with the ground node is

$$
\left(\begin{array}{ll}
G_{r r}^{\mathrm{R}} & G_{r g} \\
G_{g r} & G_{g g}
\end{array}\right)\left(\begin{array}{c}
V_{r} \\
V_{g}
\end{array}\right)=\left(\begin{array}{c}
I_{r}^{\mathrm{R}} \\
I_{g}
\end{array}\right) .
$$

The rows of (57) sum to zero so that $1 G_{r r}^{\mathrm{R}}=-G_{g r}$ and $\mathbf{1} I_{r}^{\mathrm{R}}=$ $-I_{g}$. We can choose $V_{g}=0$ in (57) and instead use $G_{r r}^{\mathrm{R}} V_{r}=$ $I_{r}^{\mathrm{R}}$. Note that $1 G_{r r}^{\mathrm{R}} \neq 0$ and $G_{r r}^{\mathrm{R}}$ is invertible. If the conductance matrix of area $\mathrm{R}$ neglecting shunts and the ground node is $G_{r r}^{\mathrm{Rnoshunt}}$, then $G_{r r}^{\mathrm{R}}=G_{r r}^{\mathrm{Rnoshunt}}-\operatorname{diag}\left\{G_{g r}\right\}$.

\section{APPENDIX C \\ EQUiVALENCE OF MulTiAREA AND Single AREA}

We show that the same voltages $V_{A}$ across area $\mathrm{R}_{\gamma}$ are obtained by simultaneous reduction of the three areas in Section VI and by the single area computation of Section IV. 
We order the interior buses in the order of the areas. Then in Section VI, the network conductance matrix becomes

$$
\begin{aligned}
G= & \left(\begin{array}{cccc}
G_{m m} & G_{m \gamma} & G_{m \alpha} & G_{m \beta} \\
G_{\gamma m} & G_{\gamma \gamma} & 0 & 0 \\
G_{\alpha m} & 0 & G_{\alpha \alpha} & 0 \\
G_{\beta m} & 0 & 0 & G_{\beta \beta}
\end{array}\right) \text { and } \\
G_{m m}^{\mathrm{red}}= & G_{m m}-G_{m \gamma} G_{\gamma \gamma}^{-1} G_{\gamma m}-G_{m \alpha} G_{\alpha \alpha}^{-1} G_{\alpha m} \\
& -G_{m \beta} G_{\beta \beta}^{-1} G_{\beta m} .
\end{aligned}
$$

In Section IV, using (21)

$$
\begin{aligned}
G_{m m}^{\mathrm{R}_{\gamma}} & =G_{m m}+\operatorname{diag}\left\{G_{m \alpha} 1\right\}+\operatorname{diag}\left\{G_{m \beta} 1\right\}, \\
G^{\mathrm{R}_{\gamma}} & =\left(\begin{array}{ll}
G_{m m}^{\mathrm{R}_{\gamma}} & G_{m \gamma} \\
G_{\gamma m} & G_{\gamma \gamma}
\end{array}\right) \\
\text { and } G_{m m}^{\mathrm{Rred}} & =G_{m m}^{\mathrm{R}_{\gamma}}-G_{m \gamma} G_{\gamma \gamma}^{-1} G_{\gamma m} .
\end{aligned}
$$

Hence

$$
\begin{aligned}
G_{m m}^{\mathrm{red}}=G_{m m}^{\mathrm{Rred}}-\operatorname{diag}\left\{G_{m \alpha} 1\right\}-G_{m \alpha} G_{\alpha \alpha}^{-1} G_{\alpha m} \\
\quad-\operatorname{diag}\left\{G_{m \beta} 1\right\}-G_{m \beta} G_{\beta \beta}^{-1} G_{\beta m} .
\end{aligned}
$$

Since

$$
\begin{aligned}
-\operatorname{diag}\left\{G_{m \alpha} 1\right\} V_{m}-G_{m \alpha} G_{\alpha \alpha}^{-1} G_{\alpha m} V_{m} \\
\quad=-\operatorname{diag}\left\{G_{m \alpha} \mathbf{1}\right\} V_{m}+G_{m \alpha} V_{\alpha}=I_{m}^{\text {ainto }}
\end{aligned}
$$

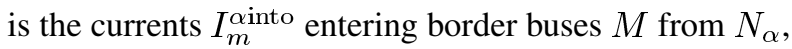

$$
\begin{aligned}
\sigma_{A} G_{m m}^{\text {red }} V_{m} & =\sigma_{A} G_{m m}^{\text {Rred }} V_{m}+\sigma_{A} I_{m}^{\text {ainto }}+\sigma_{A} I_{m}^{\text {Binto }} \\
& =\sigma_{A} G_{m m}^{\text {Rred }} V_{m}+\sigma_{A} I_{m}^{\text {into }} \\
& =\sigma_{A} G_{m m}^{\text {Rred }} V_{m}+I_{A}^{\text {into }} .
\end{aligned}
$$

Similarly

$$
\begin{aligned}
G_{A} V_{A}= & \sigma_{A} G_{m m}^{\mathrm{red}} \sigma_{A}^{T} V_{A} \\
= & \sigma_{A} G_{m m}^{\mathrm{Rred}} \sigma_{A}^{T} V_{A}-\operatorname{diag}\left\{G_{A \alpha} 1\right\} V_{A} \\
& -G_{A \alpha} G_{\alpha \alpha}^{-1} G_{\alpha A} V_{A} \\
& -\operatorname{diag}\left\{G_{A \beta} 1\right\} V_{A}+G_{A \beta} V_{A} \\
= & \sigma_{A} G_{m m}^{\text {Rred }} \sigma_{A}^{T} V_{A}+I_{A}^{\alpha \text { into }}+I_{A}^{\beta \text { into }} \\
= & \sigma_{A} G_{m m}^{\text {Rred }} \sigma_{A}^{T} V_{A}+I_{A}^{\text {into }} .
\end{aligned}
$$

Hence, $G_{A} V_{A}=\sigma_{A} G_{m m}^{\mathrm{red}} V_{m}$ is equivalent to $\sigma_{A} G_{m m}^{\mathrm{Rred}} \sigma_{A}^{T} V_{A}=\sigma_{A} G_{m m}^{\mathrm{Rred}} V_{m}$, and both equations give the same voltages $V_{A}$.

\section{APPENDIX D}

\section{PARAMETERS OF 39-Bus New ENGLAND TeSt System}

For the DC power flow, the bus power injections and bus angles are $P=0,0,-3.22,-5 ., 0,0,-2.338,-5.22,0$, $0,0,-0.085,0,0,-3.2,-3.294,0,-1.58,0,-6.8,-2.74$,
$0,-2.475,-3.086,-2.24,-1.39,-2.81,-2.06,-2.835$, $2.5,5.6373,6.5,6.32,5.08,6.5,5.6,5.4,7.81841,-0.98271$ per unit and $\theta=1.622,4.290,1.125,0.2740,1.499,2.218$, $-0.02249,-0.5267,-0.2148,4.688,3.835,3.782,3.940$, $2.219,1.794,3.334,2.188,1.323,8.473,7.113,5.923,10.81$, $10.58,3.486,5.418,3.809,1.785,7.103,9.934,6.882,10.29$, $12.14,13.61,12.35,16.13,19.31,12.60,16.92,0$ degrees. The start and end buses of the lines are 1-2, 1-39, 2-3, 2-25, $3-4,3-18,4-5,4-14,5-6,5-8,6-7,6-11,7-8,8-9,9-39$, $10-11,10-13,13-14,14-15,15-16,16-17,16-19,16-21$, $16-24,17-18,17-27,21-22,22-23,23-24,25-26,26-27$, $26-28,26-29,28-29,2-30,31-6,10-32,12-11,12-13$, 19-20, 19-33, 20-34, 22-35, 23-36, 25-37, 29-38 and the line reactances are $X=0.0411,0.025,0.0151,0.0086,0.0213$, $0.0133,0.0128,0.0129,0.0026,0.0112,0.0092,0.0082$, $0.0046,0.0363,0.025,0.0043,0.0043,0.0101,0.0217,0.0094$, $0.0089,0.0195,0.0135,0.0059,0.0082,0.0173,0.014,0.0096$, $0.035,0.0323,0.0147,0.0474,0.0625,0.0151,0.0181,0.025$, $0.02,0.0435,0.0435,0.0138,0.0142,0.018,0.0143,0.0272$, $0.0232,0.0156$ per unit. For the AC power flow, see [12] and set the transformer tap ratios to unity.

\section{REFERENCES}

[1] W.-K. Chen, Graph Theory and Its Engineering Applications. Singapore: World Scientific, 1997.

[2] I. Dobson, M. Parashar, and C. Carter, "Combining phasor measurements to monitor cutset angles," in Proc. 43rd Hawaii Int. Conf. System Sciences, Kauai, HI, Jan. 2010.

[3] I. Dobson and M. Parashar, "A cutset area concept for phasor monitoring," in Proc. IEEE PES General Meeting, Minneapolis, MN, Jul. 2010.

[4] I. Dobson, "New angles for monitoring areas," in Proc. IREP Symp., Bulk Power System Dynamics and Control_VIII, Buzios, Brazil, Aug. 2010.

[5] M. Fiedler and V. Pták, "On matrices with non-positive off-diagonal elements and positive principal minors," Czech. Math. J. vol. 12, no. 3, pp. 382-400, 1962. [Online]. Available: http://dml.cz/dmlcz/100526.

[6] F. Dörfler and F. Bullo, "Kron reduction of graphs with applications to electrical networks," eprint arXiv:1102.2950v1 [math.CO], Feb. 2011.

[7] A. G. Phadke and J. S. Thorp, Synchronized Phasor Measurements and Their Applications. New York: Springer, 2008.

[8] J. E. Tate and T. J. Overbye, "Line outage detection using phasor angle measurements," IEEE Trans. Power Systems, vol. 23, no. 4, pp. 1644-1652, Nov. 2008.

[9] R. W. Cummings, "Predicting cascading failures," presented at the NSF/EPRI Workshop Understanding and Preventing Cascading Failures in Power Systems, Westminster, CO, Oct. 2005.

[10] V. Venkatasubramanian, Y. X. Yue, G. Liu, M. Sherwood, and Q. Zhang, "Wide-area monitoring and control algorithms for large power systems using synchrophasors," in Proc. IEEE Power Systems Conf. Expo., Seattle, WA, Mar. 2009.

[11] P. R. Bryant, "The algebra and topology of electrical networks," Proc. Inst. Elect. Eng., vol. 108, pt. C, pp. 215-229, 1961.

[12] P. Ledesma, New England test system in PSS/E Format on Pablo Ledesma website. [Online]. Available: http://electrica.uc3m.es/ pablole/new_england.raw.

Ian Dobson (F'06) received the B.A. degree in mathematics from Cambridge University, Cambridge, U.K., and the Ph.D. degree in electrical engineering from Cornell University, Ithaca, NY.

He became enthusiastic about network theory when employed by EASAMS Ltd. to write a power electronic circuit simulator for the United Kingdom Atomic Energy Authority at Culham Laboratory. He previously worked for the University of Wisconsin-Madison and is currently Sandbulte Professor of Electrical and Computer Engineering at Iowa State University, Ames. 